\title{
Preventing Transient Parametric Instabilities in High Power Gravitational Wave Detectors Using Thermal Transient Compensation
}

\author{
V. Jaberian Hamedan, C. Blair, J. Liu, V. Bossilkov, C. Zhao, L. Ju, D. G. Blair \\ ARC Centre of Excellence for Gravitational Wave Discovery \\ University of Western Australia, 35 Stirling Highway, Crawley, WA 6009, Australia
}

\begin{abstract}
Laser interferometer gravitational wave detectors such as Advanced LIGO use large fused silica test masses in which temperature perturbations take many hours to reach dynamic equilibrium. When environmental disturbances cause the optical cavities to lose optical power there is a sudden thermal transient which causes the mirror profile to change significantly over time. This causes time dependent tuning of transverse optical mode frequencies, some of which cause parametric instability. These transient parametric instabilities greatly increase the complexity of active control of parametric instability. Here we report modeling and testing of a system in which a low power $\mathrm{CO}_{2}$ laser is designed to maintain a constant heat flux when cavity power is lost, thereby enabling thermal transients to be minimized and cavity locking to be re-established. We demonstrate a reduction in the thermal transient in the optical mode spacing to $<3 \%$ of the transient without compensation. For Advanced LIGO this would reduce the complexity of control systems for controlling parametric instabilities.
\end{abstract}

Keywords: Gravitational wave detectors, parametric instability, thermal transient optical mode spacing

\section{Introduction}

Three mode parametric instabilities (PI) in an optical cavity arise due to the radiation pressure mediated coupling between acoustic modes of the mirrors and cavity optical modes. In the long optical cavities of gravitational wave detectors the instabilities occur for acoustic modes in the $10-100 \mathrm{kHz}$ frequency range. PI in advanced gravitational wave detectors such as Advanced LIGO [1] and Advanced VIRGO [2] was first predicted by Braginsky et al [3] and subsequently studied by many others $[4,5,6,7]$. The phenomenon was experimentally verified in free space optical cavities in 2014 on a table top system [8] and in a suspended high power optical cavity in 2015 [9].

Parametric instability is an increasing threat as advanced detectors increase laser power to improve sensitivity to gravitational waves. Without controlling the instabilities, acoustic modes of the mirror test masses are excited to large amplitudes. At high amplitudes, the scattered light from the surface deformation of the test mass mirrors usually leads to failure of cavity locking. This has been observed in Advanced LIGO [10] and is likely to occur in Advanced VIRGO. Many parametric instability control strategies have been investigated[11, 12, 13, 14].

In optical cavities, the radius of curvature (RoC) of the mirrors is modified by optical power in the cavity, due to the residual optical absorption of the coatings. This causes a temperature gradient across the test mass, which in turn tunes the frequency of transverse optical modes that govern parametric instability. Previous studies focused on parametric instability when the cavity is in equilibrium. Here we investigate the effects of the thermal transient which develops after the cavity acquires lock. This thermal transient can result in transient parametric instability, in which a three mode interaction is unstable for a short period of time as the thermal transient evolves in the test mass.

At Advanced LIGO, when operating at $130 \mathrm{~kW}$ intracavity power $(\sim 20 \%$ of the planned design optical power), it has been observed that there are 12 transient instabilities [15] that require control as described in reference [14] to maintain interferometer operation. It is anticipated that at higher optical power many more transient instabilities will require control. Without control, the expected growth of acoustic mode amplitudes will be large. For Advanced LIGO's $40 \mathrm{~kg}$ 
fused silica test masses, it takes approximately one day for the test mass to reach a dynamic equilibrium state after a change in applied thermal load. The thermal gradient that influences the RoC becomes stable on a shorter time scale of $\sim 2$ hours. This slow tuning of the RoC gives enough time for transient unstable acoustic mode's to reach amplitudes that will disrupt the interferometer operation before the system reaches dynamic equilibrium if appropriate control is not applied. The expected amplitude transient is examined in the section on acoustic mode amplitude excursions.

This paper investigates the possibility of preventing transient instabilities. The method involves preheating the test masses with $\mathrm{CO}_{2}$ lasers so as to tune their RoCs to the anticipated "hot cavity" condition before locking the cavity. This could minimize the thermal transient, thereby stabilizing the RoC and reducing changes to the optical mode spacing. Thus transient parametric instabilities could be avoided.

The proposed laser preheating system could be important for increasing the duty cycle of GW detectors. When an earthquake or other disturbance causes the cavity to lose lock, an external heating system could be automatically activated to replace the heating due to mirror absorption. A low power $\mathrm{CO}_{2}$ laser beam for each test mass could provide the heating power. Such a system would allow the number of parametric instability control servo systems to be reduced, and the time to reestablish low noise operation may be reduced.

In this paper,we will investigate the expected amplitude excursion of an acoustic mode during transient PI in a cavity similar to Advanced LIGO. Then we will present a numerical simulation of a transient PI thermal transient compensation (TTC) scheme by preheating one mirror in an $74 \mathrm{~m}$ optical cavity with a low power $\mathrm{CO}_{2}$ laser, followed by experimental results.

\section{Theory of Parametric Instability}

In gravitational waves detector arm cavities, ideally only the fundamental mode $\left(T E M_{00}\right)$ at frequency $\omega_{0}$ is resonant. However, thermally excited test mass acoustic modes at frequency $\omega_{m}$ will scatter $T E M_{00}$ mode and create sidebands at frequencies $\omega_{0} \pm \omega_{m}$. If the sideband coincides with the cavity higher order mode $\omega_{1}$, the radiation pressure from the beating between the fundamental mode and the higher order mode acts on the acoustic mode. When the resonant condition $\omega_{0^{-}}$ $\omega_{1} \approx \omega_{m}$ is satisfied, and the spatial overlap of the optical modes and the acoustic mode is large, the 3 mode parametric interaction can become significant. The strength of the parametric interaction can be represented by the parametric gain $R_{m}$. In the case of a Fabry Pérot cavity, $R_{m}$ is defined as [16]:

$$
R_{m}=\frac{4 Q_{1} Q_{m} P}{L M c \omega_{m}^{2}} \frac{\Lambda}{\left(1+\left(\Delta \omega_{o m} / \delta_{1}\right)^{2}\right)} .
$$

Here $P$ is the power in the fundamental optical mode of the cavity; L is length of the cavity; $\mathrm{M}$ is the mass of the test mass mirror; $c$ is the speed of light. The term $\Delta \omega_{o m}=\left|\omega_{0}-\omega_{1}\right|-\omega_{m}$ defines the tuning of interaction, where $\delta_{1}$ is the optical mode linewidth. The resonant power buildup is quantified by the optical and mechanical quality factors, $Q_{1}$ and $Q_{m}$. The spatial overlap factor $\Lambda$ is used to quantify the spatial coincidence between the optical field pattern and the acoustic displacement pattern defined by [16]

$$
\Lambda=\frac{V\left(\int \Psi_{0}\left(\overrightarrow{r_{\perp}}\right) \Psi_{1}\left(\overrightarrow{r_{\perp}}\right) u_{z} d \overrightarrow{r_{\perp}}\right)^{2}}{\int\left|\Psi_{0}\right|^{2} d \overrightarrow{r_{\perp}} \int\left|\Psi_{1}\right|^{2} d r_{\perp} \int|\vec{u}|^{2} d V} .
$$

Here $\Psi_{0}$ and $\Psi_{1}$ describe the optical field distribution over the mirror surface for the fundamental mode and higher order modes respectively, $\vec{u}$ is the spatial displacement vector for the mechanical mode and $u_{z}$ is the component of $\vec{u}$ normal to the mirror surface. The integrals $\int d V$ and $\int d \vec{u}_{\perp}$ correspond to integration over the mirror volume and the mirror surface, respectively. If the parametric gain exceeds unity $(R>1)$, the acoustic mode will be excited and grow exponentially, leading to parametric instability. In general, the time constant of the acoustic mode will be changed by parametric gain;

$$
\tau_{P I}=\tau_{m} /\left(1-R_{m}\right),
$$

Here $\tau_{P I}$ is the time constant of the mode influenced by the optomechanical interaction and $\tau_{m}$ is the original time constant of the mechanical mode. From Eq. (1), it can be seen that the frequency 
resonant condition $\Delta \omega_{o m}=\left|\omega_{0}-\omega_{1}\right|-\omega_{m}$ plays an important role in the three mode interaction process, so that changes in optical mode spacing $\Delta \omega=\left|\omega_{0}-\omega_{1}\right|$ will influence the parametric gain.

Now, we will discuss how the optical mode spacing changes during a thermal transient. In a high power optical cavity, the absorbed optical power by the test mass optical coating will lead to thermal deformation of test mass mirror profile, thereby changing the frequency spacing of the fundamental mode and higher order modes, as discussed below. The optical mode spacing $\Delta \omega$ between the $T E M_{00}$ mode and $T E M_{m n}$ mode in an optical cavity is given by

$$
\Delta \omega=\frac{f_{F S R}}{\pi}(m+n) \cos ^{-1} \sqrt{\left(1-L / R_{1}\right)\left(1-L / R_{2}\right)} .
$$

Here $f_{F S R}=\mathbf{c} / 2 L$ is the free spectral range of the cavity, $\Delta \omega$ is the optical mode spacing, $\mathrm{m}$ and $\mathrm{n}$ are integer numbers that identify optical mode, $R_{1}$ and $R_{2}$ are radii of curvatures (RoC) of input test mass (ITM) and end test mass (ETM) respectively. Clearly changes of the radii of curvature $R_{1}$ and $R_{2}$ will change the mode spacing. This tuning of parametric gain by changing the frequency difference $\Delta \omega$ in Eq. 2 is the parameter of thermal tuning where a thermal flux applied to the test mass creates a thermal gradient that changes the surface profile through thermal expansion. This method was first proposed as a parametric instability control strategy [4], and has been successfully implemented $[9,10]$. When the cavities acquire lock, the optical power inside the cavities suddenly increases. Thermal heating from optical absorption of the test mass causes a growing thermal deformation of test mass which, to a good approximation, can be modeled as change in RoC of the test mass, which as discussed above, changes cavity optical mode spacing by Eq.4. As the test mass surface profile changes, the cavity mode spacing $\Delta \omega$ sweeps through a relatively large frequency range. The frequency sweep of each high order optical mode can cross many test mass acoustic modes, some of which could have $\mathrm{R}>1$. This effect can lead to transient parametric instability.

\section{Acoustic Mode Amplitude Excursion}

It was shown that operating at 10-25\% of the Advanced LIGO design power, excursions in acoustic mode amplitude of 4.5 orders of magnitude above thermally excited levels result in the interferometer losing lock ([10], [17]). Here we examine a acoustic mode growth with time due to parametric interaction. Figure 1 shows the simulation results for a $37.5 \mathrm{kHz}$ acoustic mode amplitude growth as a function of time when the cavity optical mode spacing was changing for a single cavity similar to the Advanced LIGO arm cavity [14]. The amplitude of acoustic mode is defined as $A_{m}=A_{0} e^{-t / \tau_{P I}}$. Here, it was assumed that the optical mode frequency changes at a rate of $150 \mathrm{~Hz} /$ hour which is roughly the rate for a third order optical mode in an Advanced LIGO cavity with $0.5 \mathrm{ppm}$ absorption and $120 \mathrm{~kW}$ intracavity power. The most optimistic case assumes an optical mode linewidth of $30 \mathrm{~Hz}$ like those in recent coupled cavity simulations [18] and maximum parametric gain of 3. In this case, acoustic mode amplitude excursions of 4.5 orders of magnitude are expected (curve (a)). In the worst case, maximum parametric gain of 8 and the linewidth of the single arm cavity $\sim 90 \mathrm{~Hz}$ are used. In this case the rapid increase in acoustic mode amplitude far exceeds the interferometers limits (curve (d)). The instrument would likely lose lock in 20 minutes.

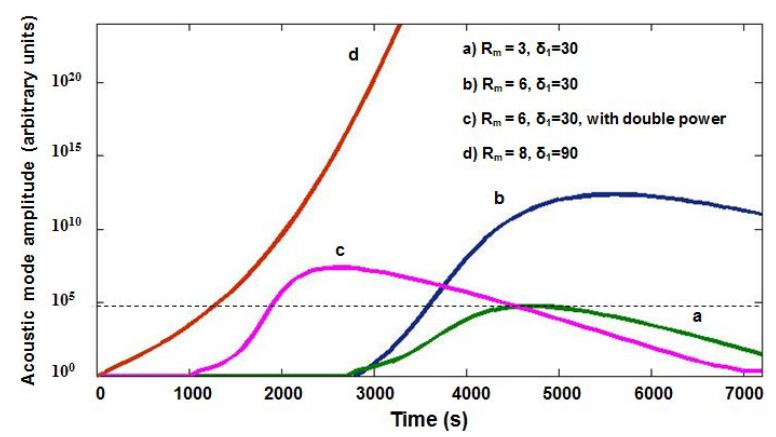

Figure 1: Simulation results of $37.5 \mathrm{kHz}$ acoustic mode amplitude excursion due to parametric interaction for a single cavity similar to Advanced LIGO's, where the optical mode frequency changes at a rate of $150 \mathrm{~Hz} /$ hour for various scenarios. The dotted line indicates the acoustic mode amplitude level, above which the interferometer would lose lock. 
It should be noted that the amplitude excursion of the acoustic mode is relatively weakly dependent on optical power compared to the gain and linewidth dependence. For example, for the same cavity configuration as the best case (a) in Figure 1, if the power level is doubled, which in effect doubled the gain $\left(R_{m}=6\right)$, the maximum excursion amplitude will increase to 7 orders of magnitude instead of 13 orders of magnitude as curve (c) with the same $R_{m}=6$ but less power. This is because at higher power, the increase in amplitude excursion due to higher $R_{m}$ is somewhat compensated by the higher rate of change of the optical mode frequency which acts to reduce the amplitude excursion. Thus, we can expect transient instabilities to induce amplitude excursions that unlock the interferometer even at a relatively lower power level than the designed power level.

\section{Thermal Transient Compensation Simulation}

A numerical finite element simulation was used to investigate thermal transient parametric instability, and the capability of compensating the thermal transient through preheating one of the test masses in a cavity using a $\mathrm{CO}_{2}$ laser.

Figure 2 shows a schematic of the thermal transient compensation (TTC) system. In the simulation, the input test mass (ITM) was preheated by a nominal constant absorbed power $P_{1}$. Then the heat load is switched from $P_{1}$ on the ITM alone to $P_{2}$ on both the ITM and ETM to reflect the locked cavity condition with the $\mathrm{CO}_{2}$ laser turned off. From this point onwards until dynamic equilibrium is reached, the ETM heats up with heat load $P_{2}$, while the ITM cools under the reduced heat load (from $P_{1}$ to $P_{2}$ ).

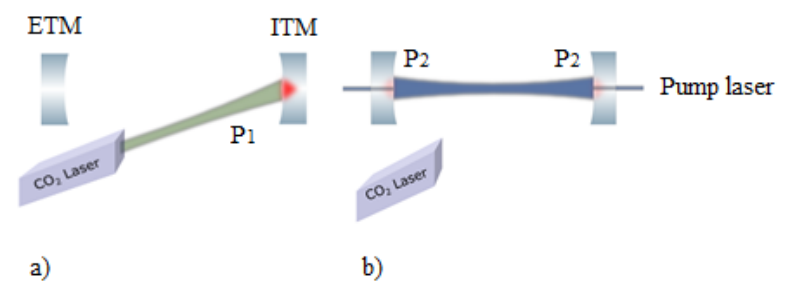

Figure 2: Thermal transient compensation system scheme; a) $\mathrm{CO}_{2}$ laser preheating ITM with power $P_{1}$ when the cavity is not locked; b) When the cavity is locked, the self-heating dissipates power $P_{2}$ in both mirrors. The $\mathrm{CO}_{2}$ laser is simultaneously turned off.

The time dependence of the surface deformation of the two test masses was obtained from finite element modelling using COMSOL [19] Heat Transfer in Solids with Thermal Expansion analysis. From COMSOL generated surface deformation maps the FFT based optical simulation tool, OSCAR [20] was used to estimate the change in RoC of each test mass. The area used for the RoC estimation was defined by the $1 / e^{2}$ radius of the cavity circulating beam. The mode spacing over time was calculated using Eq. (4) (and was verified with OSCAR). Here we only consider the $T E M_{01}$ higher order mode, so that $(m+n)=1$ in Eq. (4). We used the cavity parameters of the $74 \mathrm{~m}$ suspended cavity at the Gingin High Optical Power Facility [21] in this simulation, as shown in Table 1.

\begin{tabular}{l|l} 
Cavity Length $(\mathrm{m})$ & 74 \\
\hline Cold cavity ITM RoC $(\mathrm{m})$ & 37.20 \\
\hline Cold cavity ETM RoC $(\mathrm{m})$ & 37.16 \\
\hline ITM dimensions $(\mathrm{m})$ & $\varnothing 0.1 \times 0.05$ \\
\hline ETM dimensions $(\mathrm{m})$ & $\varnothing 0.1 \times 0.05$ \\
\hline $\begin{array}{l}\text { Heat load on both ITM and ETM mimicking absorbed of main } \\
\text { cavity laser } P_{2}(\mathrm{~W})\end{array}$ & 0.055 \\
\hline Heating $P_{2}$ spot size $(\mathrm{m})$ & 0.0114
\end{tabular}

Table 1: Simulation parameters

We compared situations with various preheating powers and spot sizes. Figure 3 shows the simulated RoC of the ITM and ETM changing as a function of time. Time $t=0$ represents the 
time when the cavity acquires lock and the $\mathrm{CO}_{2}$ heating (if present) is switched off. It is assumed that the ITM has reached equilibrium with $\mathrm{CO}_{2}$ heating prior to $t=0$. The $37 \mathrm{~m}$ RoC of the ETM is changed by $\sim 50 \mathrm{~cm}$ due to absorbed power $P_{2}=0.055 \mathrm{~W}$. Consider the case where the $\mathrm{CO}_{2}$ preheating spot size is the same as the cavity beam size and the preheating power $P_{1}$ is double the absorbed power $P_{2}$, i.e. $P_{1}=2 P_{2}$. In this case the rate of the RoC change of the two test masses will be similar, but with the opposite sign. However, if the preheating spot size was different from the cavity beam size, the balance between the two RoC change would not occur because the rate of deformation depends on both power and spot size. Thus, there would be a mismatch of the RoC rate of change between the two test masses.

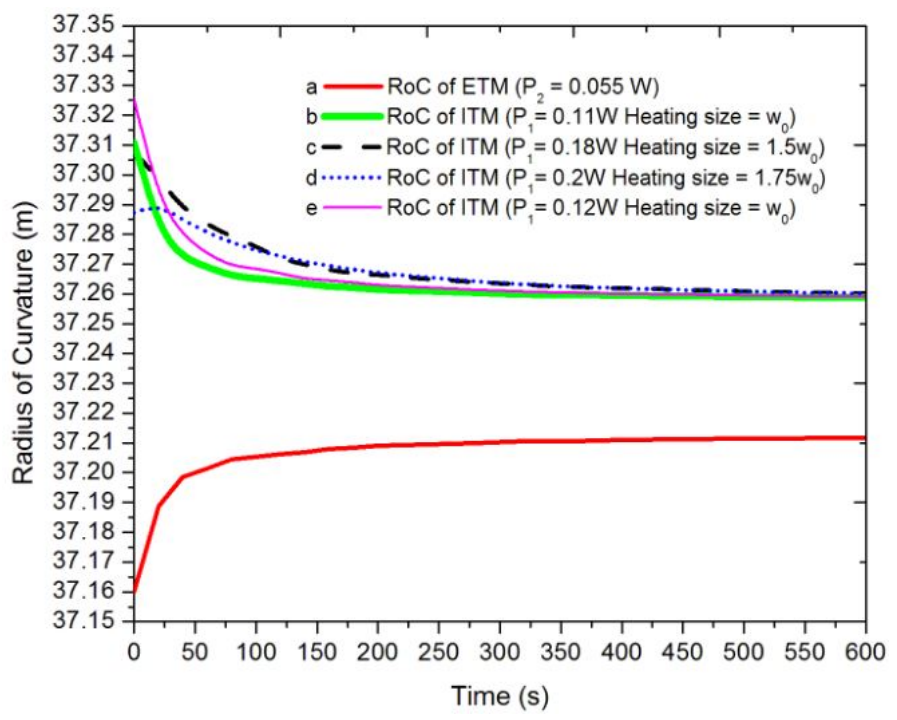

Figure 3: The simulation results of the ITM and ETM radius of curvature (RoC) changing with time after the cavity is locked, with absorbed power of $0.055 \mathrm{~W}$ (beam size $w_{0}=0.0114 \mathrm{~m}$ ), and with different preheating on ITM. Time $t=0$ represents the moment when the cavity is locked and the preheating is turned off. (a) Bottom red solid line: changing in the RoC of the ETM as it self-heats. (b) Thick green solid line: ITM RoC change as it cools after being preheated with $0.11 \mathrm{~W}$ and spot size $w=w_{0}$. It almost matches the heating rate of the RoC of the ETM. Curves (c), (d) and (e) show the effect RoC of the ITM with different unmatched spot sizes and heating. (c) Dashed line: preheating $0.18 \mathrm{~W}$ and spot size $w=1.5 w_{0}$; (d) Dotted line: Preheating $0.2 \mathrm{~W}$ and spot size $w=1.75 w_{0}$; (e) thin solid crayon line: preheating $0.12 \mathrm{~W}$ and spot size $w=w_{0}$.

For this reason, the preheating load and spot size needs to be adjusted so as to minimize the difference between ITM and ETM RoC change rate. Figure 2 shows the RoC change of the ITM and ETM after the cavity is locked with $0.055 \mathrm{~W}$ of absorbed power, with different preheating on the ITM.

Figure 4 shows the cavity mode spacing between $T E M_{00}$ and $T E M_{01}$ mode as a function of time with and without preheating. It can be seen that, without preheating, both test masses have the same heat load of $P_{2}$ starting at $t=0$, the mode spacing swept through $\sim 12 \mathrm{kHz}$. In the case where preheating spot size equals main cavity spot size and the preheating power is double the main cavity absorbed power, the ETM self-heating is effectively compensated by the cooling effect on the ITM. The mode spacing changing is very small (about $70 \mathrm{~Hz}$ - green thick line) due to the similar and opposite RoC change of the two test masses as shown in Fig. 3. Increasing the preheating power by $10 \%$, causes the mode spacing change to increase to about $1.5 \mathrm{kHz}$. If the spot sizes are not matched, the RoC changes rates no longer match, so that it is impossible to achieve good compensation over all these. Typically, there is a period $\sim 5$ minutes over which the mode spacing varies from $\sim 2 \mathrm{kHz}$ to a few hundred $\mathrm{Hz}$.

\section{Experimental Results}

Experimental confirmation of the above modelling was carried out using a $74 \mathrm{~m}$ high power suspended cavity. A $50 \mathrm{~W}$ fiber amplifier pumped by an NPRO is frequency locked to the suspended 


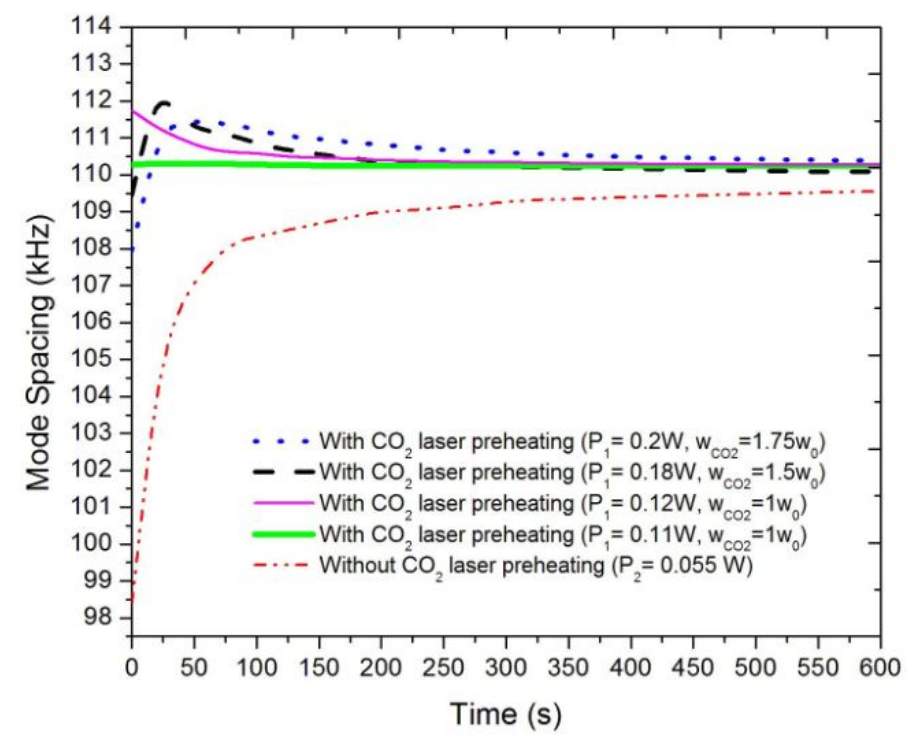

Figure 4: Simulation results of the mode spacing transients of a cavity with and without preheating on ITM. Dashed-dotted red line - no pre-heating; Green thick solid line - ideal pre-heating: preheating ITM with double the cavity absorbed power $\left(P_{1}=2 P_{2}\right)$ and same heating spot size as cavity laser size $\left(w_{0}\right)$; Purple thin solid line - with $P_{1}=2.18 P_{2}$ and $w=w_{0}$; Black dashed line - with $P_{1}=3.36 P_{2}$ and $w=1.5 w_{0}$; Blue dotted line - with $P_{1}=3.63 P_{2}$ and $w=1.75 w_{0}$.

cavity using the Pound-Drever-Hall (PDH) technique. The output of the fiber amplifier is filtered with a pre-mode cleaner before injection into the cavity. The cavity has a finesse of 15,000 and $\mathrm{g}$-factor of $\sim 0.96$. With this configuration it is possible to observe the high order transverse optical modes beating with the fundamental mode as resonant peaks in the transmitted beam. The concept of the experiment is to preheat ITM with a $\mathrm{CO}_{2}$ laser prior to locking the cavity. When the cavity is locked the $\mathrm{CO}_{2}$ laser is turned off simultaneously. The cooling of the ITM then compensates for the heating of the ITM as shown in the simulation in the previous section. The required $\mathrm{CO}_{2}$ power was determined empirically by first measuring the transient in the optical mode spacing due to the laser power heating in the cavity. Then with very low optical power for locking the cavity, the transient in the mode spacing was measured as a function of $\mathrm{CO}_{2}$ laser power on the ITM. The $\mathrm{CO}_{2}$ laser power that produced the same mode spacing change as that with the full cavity power was then used as the thermal compensation power $P_{2}$. With almost no power in the cavity the (cold cavity) mode spacing was measured to be $98.2 \mathrm{kHz}$. When the intracavity power is $6 \mathrm{~kW}$, the (hot cavity) mode spacing increases to $109.8 \mathrm{kHz}$. Therefore, the mode spacing transient is $\sim 12 \mathrm{kHz}$ (as we will see in Fig. 6). From this transient, the coating absorption was estimated to be $9 \pm 0.7 \mathrm{ppm}$, assuming equal absorption on each test mass. With very little intracavity power the mode spacing was recorded as a function of $\mathrm{CO}_{2}$ laser power as shown in Fig. 5. It was found that a $\mathrm{CO}_{2}$ laser power of $\sim 1.8 \mathrm{~W}$ resulted in a stable mode spacing of $109.8 \pm 0.5 \mathrm{kHz}$. The $\mathrm{CO}_{2}$ laser beam size on the ITM was estimated to be $\sim 1.5$ times of the main cavity beam size. A simulation with these parameters is in agreement with the measurement for $0.18 \mathrm{~W} \mathrm{CO}_{2}$ laser power. Deviation from the simulation at higher and lower power are not well understood. Based on the measurement, we chose a power of $\mathrm{CO}_{2}$ laser power of $0.18 \mathrm{~W}$ to perform the experiment.

The compensation scheme applied $0.18 \mathrm{~W}$ of $\mathrm{CO}_{2}$ laser power to the ITM before locking the cavity. A preheating time $\sim 2$ minutes was used to achieve dynamic thermal equilibrium. Then the cavity was locked and the $\mathrm{CO}_{2}$ laser turned off. Figure 6 shows the measured mode spacing change as a function of time with (green points) and without (red points) preheating. It can be seen that without any preheating, the cavity mode spacing swept through $\sim 12 \mathrm{kHz}$ in 2 minutes, while, with preheating, the mode spacing stayed almost constant. The $\sim 3 \%$ variation in mode spacing with respect to the uncompensated system is within measurement error of the ideally compensated system with no change in mode spacing.

The experimental result is surprisingly flat when compared to the simulation (black curve Fig. 6) for $\mathrm{CO}_{2}$ preheating with $0.18 \mathrm{~W}$ and beam size of $1.5 w_{0}$ which predicts a mode spacing change of about $2.5 \mathrm{kHz}$. A likely explanation is that relatively large residual motion of the cavity 


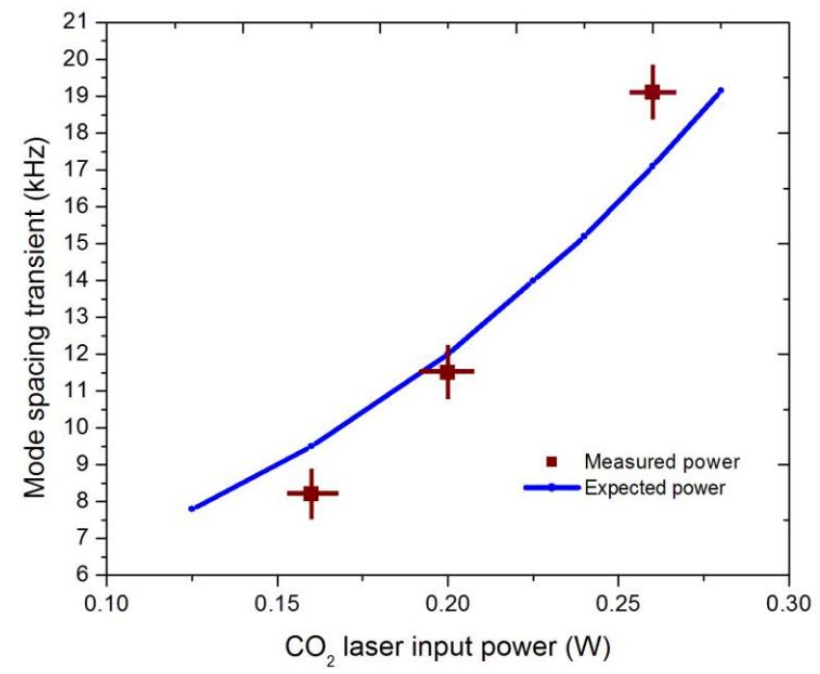

Figure 5: Experimental results of mode spacing transients by $\mathrm{CO}_{2}$ laser heating ITM. Solid line shows the expected mode spacing change as a function of absorbed power at ITM.

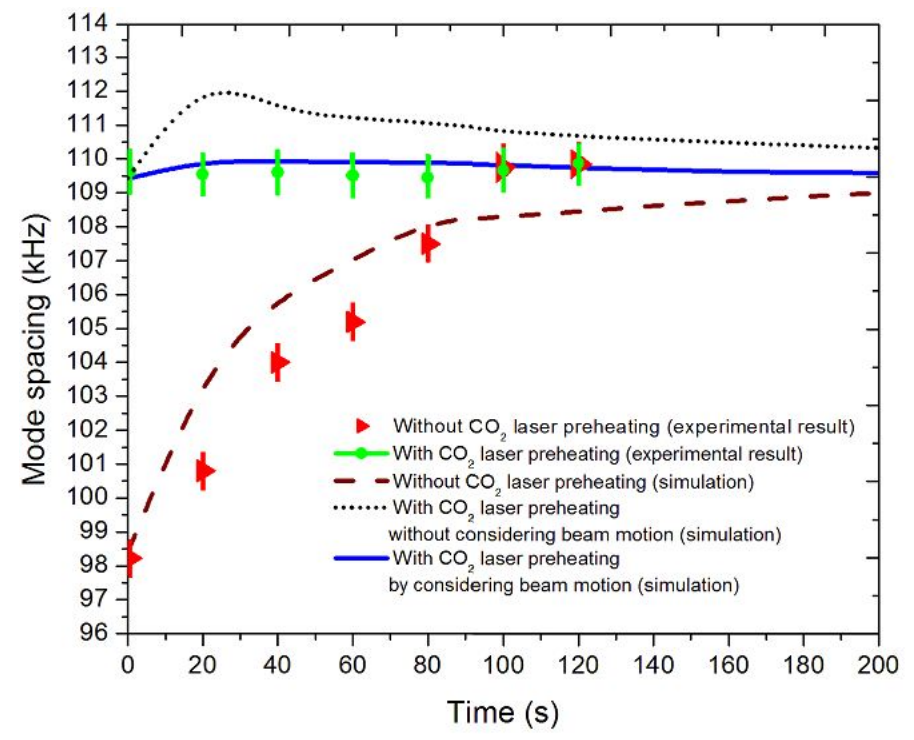

Figure 6: Experimental results on transient cavity mode spacing with and without preheating. Red triangle: Without preheating, the cavity mode spacing changed about $12 \mathrm{kHz}$ due to the absorption of the cavity laser power by both ITM and ETM. Green dots: with preheating on ITM. For comparison, some simulation results are also shown. Dashed line: simulation result of cavity transient when both mirror absorb $10 \mathrm{ppm}$ of $6 \mathrm{~kW}$ laser power, without preheating. Black dotted line: simulation with preheating ITM by $0.18 \mathrm{~W}$ power and beam size of $1.5 w_{0}$; Blue solid line: simulated result by preheating the ITM with a cluster of four $0.18 \mathrm{~W}, 1.5 w_{0}$ beam size to mimic the average heating area due to the mirror motion

beam on the test mass changes the effective self-heating pattern. This beam motion is caused by mirror residual motion at suspension resonant frequency $\sim 1 \mathrm{~Hz}$. To simulate this we consider a time averaged heating pattern, where the heating beam moves by half the heating beam radii peak to peak in the vertical and horizontal planes. This time averaged heating pattern produces a mode spacing transient (blue curve) that is consistent with the experimental results.

\section{Discussion}

A thermal transient compensation (TTC) scheme for eliminating transient PI in a $74 \mathrm{~m}$ optical cavity has been investigated. $\mathrm{CO}_{2}$ laser preheating on just one cavity test mass mirror enabled 
the self-heating transient in the first transverse optical mode frequency to be stabilized within experimental error, which was less than $3 \%$ of its uncompensated transient. We find that such a scheme which allows one test mass to be heated while the other cools is adequate for nulling the transient in the optical mode spacing. This is due to the convenient fact that the rate of change in $\mathrm{RoC}$ of the two test masses is proportional to the change in heating power.

If the cavity mirrors' RoCs, emissivities or absorptions were not the same, single mirror heating may not result in a very good transient compensation. Further investigation is required to determine whether using a single mirror heating with some optimised heating spot size and power level could still result in a good thermal transient compensation.

Clearly the best solution for transient PI would be to use a TTC system for each test mass of the arm cavities. This can maintain approximately constant temperature profiles in all test masses minimising the change in acoustic mode frequencies and also prevent time dependent wavefront distortions in the reflected beams that cause changes in the optical eigenfrequencies. In this case to achieve an effective nulling of the thermal transient, the $\mathrm{CO}_{2}$ heating beams need to match the spot size, and the absorbed power of the main cavity laser on each individual test mass.

Our modelling also showed that if the preheating spot size is 1.5 times that of the main cavity laser spot size, it is possible to reduce the thermal transient in mode spacing change to $\sim 10 \%$ of the uncompensated transient by adjusting the $\mathrm{CO}_{2}$ laser power appropriately. Thus some errors in spot size can be tolerated. The required precision of the TCC will depend on the number of transient parametric instabilities at a particular power and their parametric gain.

The TTC scheme could help to improve detector operation duty cycle by avoiding occasional lock lost due to transient PIs and reduce the detector complexity by reducing the number of control servos required to suppress PI. Since $\mathrm{CO}_{2}$ preheating would not be present during sensitive operation of the gravitational wave detector, the noise requirement of the preheating system is not stringent. However $\mathrm{CO}_{2}$ laser power stability and changes of ambient temperature which affect the radiation heat loss of the test masses could set limits on the TTC system performance.

Another issue identified in this paper that could be relevant to implementing a TTC is the residual motion of the beam position on the test mass. This was shown to change the effective heating pattern and time constant. Long term drifts in beam position between one lock and the next would result in a change in position of the heat load. In addition, in the experiment reported here the $\mathrm{CO}_{2}$ laser heats the test mass at a significant oblique angle. Although it did not affect our proof of principle result, the associated astigmatism of the heating spot needs to be considered in designing the TTC system.

Finally technical aspects such as the speed at which the $\mathrm{CO}_{2}$ laser can automatically adjust for changes in cavity power during the power up sequence and when cavity lock is lost may also lead to transients in thermal load. A careful analysis of many such subtle effects is required.

Further work is needed to fully model the thermal transient PI and TTC system for Advanced LIGO arm cavities.

\section{Acknowledgement}

The authors would like to thank the LIGO Scientific Collaboration (LSC) for reviewing this paper and useful suggestions. This work is supported by Australian Research Council.

\section{References}

[1] J Aasi et. al. (LIGO Scientific Collaboration). Advanced ligo. Classical and Quantum Gravity, $32(7), 2015$.

[2] F Acernese et. al. Virgo: A large interferometer for gravitational wave detection started its first scientific run. Journal of Physics: Conference Series, 120(3), 2008.

[3] S. Vyatchanin Braginsky, V. Strigin. Parametric oscillatory instability in fabry-perot interferometer. Phys. Lett. A, 287(94), Apr 2001.

[4] C. Zhao, L. Ju, J. Degallaix, S. Gras, and D. G. Blair. Parametric instabilities and their control in advanced interferometer gravitational-wave detectors. Phys. Rev. Lett., 94(12), Apr 2005. 
[5] Zhao C. Gras S.M. Degallaix J. Blair D.G. Munch J. Ju, L. and D.H Reitze. Comparison of parametric instabilities for different test mass materials in advanced gravitational wave interferometers. Phys. Lett. A, 355(6), Apr 2006.

[6] I.A. Polyakov and S.P. Vyatchanin. Precursors of parametric oscillatory instability in $\{$ LIGO $\}$ interferometer. Physics Letters A, 368(6), 2007.

[7] Li Ju, Chunnong Zhao, Yiqiu Ma, David Blair, Stefan L Danilishin, and Slawek Gras. Three mode interaction noise in laser interferometer gravitational wave detectors. Classical and Quantum Gravity, 31(14), 2014.

[8] Chen X et. al. Observation of three-mode parametric instability. Phys. Rev. A, 91(3), Mar 2015.

[9] Chunnong Zhao, Li Ju, Qi Fang, Carl Blair, Jiayi Qin, David Blair, Jerome Degallaix, and Hiroaki Yamamoto. Parametric instability in long optical cavities and suppression by dynamic transverse mode frequency modulation. Phys. Rev. D, 91(9), May 2015.

[10] Evans et.al. Observation of parametric instability in advanced ligo. Phys. Rev. Lett., 114(16), Apr 2015.

[11] S Gras, P Fritschel, L Barsotti, and M Evans. Resonant Dampers for Parametric Instabilities in Gravitational Wave Detectors. Phys. Rev., D92(8), 2015.

[12] Miller J et. al. Damping parametric instabilities in future gravitational wave detectors by means of electrostatic actuators. Phys. Lett., A375, 2011.

[13] Andrey B. Matsko, Mikhail V. Poplavskiy, Hiroaki Yamamoto, and Sergey P. Vyatchanin. Mitigating parametric instability in optical gravitational wave detectors. Phys. Rev. D, 93(8), Apr 2016.

[14] C. Blair et al. First demonstration of electrostatic damping of parametric instability at advanced ligo. Physics Review Lett., 118(15), Apr 2017.

[15] Jenne Driggers et al. Commissioning progress and plans at lho - lvc meeting. document number G1700315, https://dcc.ligo.org/DocDB/0140/G1700315/001/H1CommishUpdate.pdf, 2017.

[16] S. P. Vyatchanin V. B. Braginsky, S. E. Strigin. Analysis of parametric oscillatory instability in power recycled ligo interferometer. Phys.Lett. A, 305, 2002.

[17] D. Blair et al. The next detectors for gravitational wave astronomy. Science China Physics, Mechanics and Astronomy, 58(12), 2015.

[18] M. Dovale-Álvarez C. Collins H. Miao C. Mow-Lowry A. Freise A. C. Green, D. D. Brown. The influence of dual-recycling on parametric instabilities at advanced ligo. arxiv e-print, 1704(08595), 2017.

[19] COMSOL. version 4.4. COMSOL Inc., Burlington, Massachusetts, USA, 2013.

[20] Jérôme Degallaix. Oscar a matlab based optical fft code. Journal of Physics: Conference Series, 228(1), 2010.

[21] C Zhao et al. Gingin high optical power test facility. Journal of Physics: Conference Series, $32(1), 2006$. 